\title{
Stability properties of phase transition layers in the diffuse ISM revisited
}

\author{
Jennifer M. Stone ${ }^{1}$, Shu-ichiro Inutsuka ${ }^{1}$, \\ and Ellen G. Zweibel ${ }^{2}$
}

${ }^{1}$ Department of Physics, Nagoya University, Furo-cho, Chikusa-ku, Aichi 464-8602, Japan email: stone@nagoya-u.jp

${ }^{2}$ Departments of Phyics and Astronomy and Center for Magnetic Self-Organization, University of Wisconsin-Madison, 475 N Charter Street, Madison, WI 53706, USA

\begin{abstract}
In a thermally bistable medium, cold, dense gas is separated from warm, rarified gas by thin phase transition layers, or fronts, in which radiative heating/cooling, thermal conduction, and convection of material are balanced. While these fronts have received only scant attention in the literature, and are not resolved by most current numerical simulations, they have been shown to have important ramifications for transport processes and structure formation in the diffuse interstellar medium. Here, we discuss calculations of their hydrodynamic and magnetohydrodynamic stability properties.
\end{abstract}

Keywords. hydrodynamics, ISM: structure, instabilities, (magnetohydrodynamics:) MHD, methods: numerical

The corrugational instability in phase transtion layers is an intriguing mechanism for sustaining turbulence (e.g. Koyama \& Inutsuka 2002) and generating small scale structure (e.g. Heiles 1997) in the diffuse interstellar medium. Previous attempts at calculating the full hydrodynamic stability behavior of thermal fronts have encountered mathematical difficulties in that conventional methods for solving eigenvalue problems do not converge. As a result, all prior investigations have relied upon simplifying approximations to make the calculation more tractable (Inoue et al. 2006). However, while such approaches enable conceptual progress, they do not allow for an accurate description of the overall hydrodynamic stability properties of thermal fronts. Furthermore, corresponding approximations are not valid in the magnetohydrodynamic regime. Work is ongoing to develop a numerical method to calculate the full hydrodynamic stability properties of thermal fronts, without the need for approximation. Ultimately, we will extend these efforts to study the effects of magnetic fields and ambipolar diffusion (Stone \& Zweibel 2009, 2010) on phase transition layer stability.

\section{Acknowledgements}

J.M.S. gratefully acknowledges support from the Global COE Program "Quest for Fundamental Principles in the Universe: from Particles to the Solar System and Cosmos" of Nagoya University.

\section{References}

Heiles, C. 1997, ApJ 481, 193

Inoue, T., Inutsuka, S.-i., \& Koyama, H. 2006, ApJ 652, 1331

Koyama, H. \& Inutsuka, S.-i. 2002, ApJ (Letters) 564, L97

Stone, J. M. \& Zweibel, E. G. 2009, ApJ 696, 233

Stone, J. M. \& Zweibel, E. G. 2010, ApJ 724, 131 